\title{
OCT Glaucoma Staging System: a new method for retinal nerve fiber layer damage classification using spectral-domain OCT
}

P Brusini

\begin{abstract}
Purpose To describe a new method, the Optical Coherence Tomography (OCT) Glaucoma Staging System, for classifying retinal nerve fiber layer (RNFL) damage

practice for an easy and fast interpretation of RNFL measurements obtained with OCT. Eye (2018) 32, 113-119; doi:10.1038/eye.2017.159; published online 4 August 2017
\end{abstract} assessed with OCT.

Patients and methods The OCT Glaucoma Staging System was created based on data obtained from Nidek RS 3000 spectraldomain (sd)-OCT. This system uses the superior and inferior quadrant RNFL thickness values, plotted on an $x-y$ diagram for staging structural damage severity in glaucoma. A non-linear equation and two regression lines describe the boundary lines which separate the different sectors of the diagram. These mathematical formulas have been used to create a software, which provides a quick classification of the RNFL damage. Sensitivity and specificity of the system were assessed in a different cohort including 64 patients with early OAG, and 62 normal subjects.

Results Three hundred and two OCT tests from 98 healthy controls and 284 patients affected by either ocular hypertension or chronic open-angle glaucoma were considered in order to design the new classification system. The OCT Glaucoma Staging System classifies RNFL defects into 6 stages of increasing severity ranging from borderline to stage 5 , and 3 groups according to defect localization (superior, inferior, or diffuse). Sensitivity and specificity in discriminating between healthy and glaucomatous eyes were 95.2 and $91.9 \%$, respectively, considering borderline results as abnormal.

Conclusions The OCT Glaucoma Staging System appears to provide a standardized and objective classification of glaucomatous RNFL damage. It can be used in day-to-day clinical

\section{Introduction}

Optical Coherence Tomograpy (OCT) is a noninvasive imaging technique designed to quantitatively assess the macular retinal layers and the peripapillary retinal nerve fiber layer (RNFL) in vivo. ${ }^{1-3}$ The new spectral-domain (sd) OCTs allow for a more accurate and reproducible measurement of RNFL thickness, thus providing high diagnostic ability in discriminating between healthy and glaucomatous eyes. ${ }^{4-14}$

All current OCT instruments provide quite similar test result representation, which includes numerical data, graphs, and color-coded maps that aid in the interpretation of data. Early defects, however, may be missed, especially by ophthalmologists with limited experience in glaucoma management. Moreover, the currently available instruments do not provide a classification of the severity of RNFL loss, similarly to methods available for visual field defect severity staging ${ }^{15}$ thus subjective criteria must be used. At present, the RNFL loss only assessed with Scanning Laser Polarimeter with Variable Corneal Compensator (GDx VCC) can be staged using the GDx Staging System. ${ }^{16}$

The purpose of this study was to introduce a new, simple, and easy-to-use method, called the OCT Glaucoma Staging System (OCT GSS), which plots two OCT parameters on an $x-y$ diagram to simultaneously provide both a standardized classification of RNFL defect severity and the defect localization.
Department of

Ophthalmology - Policlinico, 'Città di Udine', Udine, Italy

Correspondence:

P Brusini, Department of Ophthalmology - Policlinico 'Città di Udine', Viale Venezia 410, Udine 33100, Italy

Tel: +432-239371;

Fax: +432-545400.

E-mail: brusini@libero.it

Received: 14 September 2016

Accepted in revised form:

4 May 2017

Published online:

4 August 2017 


\section{Material and methods}

\section{Patients}

The study included 284 patients affected by either ocular hypertension $(\mathrm{OH})$ (intraocular pressure (IOP) $>21 \mathrm{~mm} \mathrm{Hg}$; normal optic nerve head $(\mathrm{ONH})$ and RNFL appearance, and normal visual field on standard automated perimetry (SAP)) or chronic open-angle glaucoma (OAG) (IOP > $21 \mathrm{~mm} \mathrm{Hg}$ before medication, abnormal slit-lamp appearance of the ONH or RNFL, and reproducible glaucomatous visual field defects); and 98 normal subjects (IOP, ONH, RNFL, and SAP within normal limits; no family history of glaucoma and other ocular pathologies).

Only one eye per subject was randomly selected if both eyes met the inclusion criteria. All subjects underwent a complete ophthalmologic examination, including SAP, GDx VCC, Heidelberg Retina Tomograph (HRT) and sdOCT testing within a 3-month period. Normal subjects were recruited from staff members and volunteers. $\mathrm{OH}$ and OAG patients were recruited from the Glaucoma Center of the Department of Ophthalmology at the Hospital of Udine, Italy. The study was in compliance with the tenets of the Helsinki's Declaration, and with Institutional Review Boards (IRBs) requirements for our institute. Informed consent was obtained from all participants prior to testing.

Inclusion criteria were: best corrected visual acuity better than or equal to 0.7 ; absence of ocular pathology other than open-angle glaucoma; reliable SAP test results; and good OCT image quality.

Exclusion criteria included: ametropia $> \pm 5$ diopters, pupil diameter $<2 \mathrm{~mm}$; secondary causes of glaucoma; papillary anomalies; large peripapillary atrophy; media opacities preventing good image scans; and neurological disorders.

\section{Methods}

IOP was measured with Goldmann applanation tonometry.

$\mathrm{ONH}$ was assessed by a glaucoma expert ophthalmologist with slit-lamp stereoscopic ophthalmoscopy using a 78-diopter lens.

SAP was performed using either the Humphrey Field Analyzer (HFA) II 750 (Carl Zeiss Meditec Inc., Dublin, California) 24-2 or 30-2 test with Standard Swedish Interactive Thresholding Algorithm (SITA) strategy. SAP tests were classified as glaucomatous following the Anderson and Patella criteria. ${ }^{17}$ The severity of visual field defects was classified using the Glaucoma Staging System 2 (GSS 2). ${ }^{18}$

OCT was performed with the Nidek RS-3000 Advance Capture sd-OCT (Nidek, Gamagori, Japan; software version Navis-Ex 1.4.0.1). The RNFL thickness measurements were performed using the Disc Map protocol $(6 \times 6 \mathrm{~mm})$. Only reliable results (signal strength index $\geq 7 / 10$ with no evident artifacts) were considered in the analysis.

The aging effect on RNFL thickness was taken into account, according to normative database of Nidek RS-3000 OCT, considering a $0.3 \mu$ decrease per year, with a larger decrease in the superior quadrant and in patients over the age of 50 years.

The differences amongst the currently available OCT devices on the market were also taken into account, using our previous unpublished clinical data and the tables published by Pierro et al ${ }^{19}$ which list the differences in RNFL thickness for seven different OCT instruments.

\section{Data processing and algorithm development}

The OCT Glaucoma Staging System uses both the Superior Quadrant Average and the Inferior Quadrant Average values, taken from the Temporal-SuperiorNasal-Inferior-Temporal (TSNIT) map, plotted on an $x-y$ axes diagram, respectively (Figure 1). Other parameters, such as whole RNFL thickness, Disc Area, and Cup Area, previously considered (data not shown), did not offer comparable reliability in classifying the structural damage. Six curve lines divide the diagram into 7 sectors: normal RNFL results are displayed in the superior-left corner of the graph, while RNFL defects are classified into 6 stages of increasing severity, from borderline to stage 5 (almost complete loss of RNFL).

The normative data provided by the sd-OCT Nidek RS-3000 were used to plot both the curvilinear lines that divide the normal results from borderline stage, and the borderline results from abnormal data, respectively. In order to divide RNFL defects in the stages of damage severity ranging from 1 to 5 , an arbitrary subdivision was utilized, based on structural glaucomatous damage assessed with clinical optic disc assessment, using the Optic Disc Damage Staging System (ODDSS), ${ }^{20}$ and data from both GDx VCC and Heildelberg Retina Tomograph.

Moreover, two linear regression lines divide the diagram in three sectors: RNFL defects mostly localized in the superior quadrant are found in the upper right portion of the graph, while defects mostly localized in the inferior quadrant are found in the bottom left portion of the graph. Defects that affect both these quadrants are shown in between and labeled as diffuse defects. A defect was considered to affect either the superior or the inferior sector when a clear asymmetry in the normative database and/or TSNIT maps was present. In the remaining cases the defect was defined as diffuse. 




Figure 1 Graphical representation of the OCT Glaucoma Staging System in a 61-year-old patient with POAG in the left eye. The intersection of the superior and inferior quadrant RNFL thickness values (arrows), expressed in microns, defines both the stage and the prevalent localization of the retinal nerve fiber defect.

The mathematical formulas reported in the results section were used to create a software, in order to provide a quick classification of the RNFL damage.

\section{Validation of the OCT Glaucoma Staging System}

In order to validate the OCT Glaucoma Staging System, its sensitivity and specificity in discriminating between healthy and glaucomatous subjects were assessed in a different cohort, including 64 patients with early chronic OAG (GSS 2 stages 1 or 2), and 62 normal subjects. The inclusion and exclusion criteria, testing methods and OCT RNFL analysis protocol were identical to those used in the first part of the study. In this cohort, however, eyes with advanced glaucomatous damage were excluded to avoid reporting an artful increase in the sensitivity of this new method.

\section{Statistical analysis}

Linear regression analysis, where $y=a x-b$, was used to calculate the two lines dividing the OCT Staging System diagram in 3 sectors. The coefficients of the 6 curves dividing the OCT Glaucoma Staging System diagram in 7 stages were calculated using the non-linear regression analysis and expressed by the following formula: $\alpha x+\beta x^{2}+\gamma$.

The statistical analysis was performed using SPSS 11.0 for Windows (SPSS Inc, Chicago, IL, USA). Statistical significance was defined as $P<0.5$.

\section{Results}

The study included 94 patients with $\mathrm{OH}, 190$ patients affected by OAG and 98 normal subjects as a control group. The OCT results obtained from 4 OAG patients were excluded because of poor image quality. A total of 378 sd-OCT tests from $94 \mathrm{OHT}$ patients (mean age $61.3 \pm 11.7$; range $37-83$ ); 186 OAG patients (mean age 67.1 \pm 16.4; range 35-91; mean SAP-MD: $-8.7 \mathrm{~dB} \pm 7.1$; range -0.35 to $-29.6 \mathrm{~dB}$ ), and 98 healthy controls (age $56.6 \pm 13.8$; range $31-83$ ) was used to create the OCT Glaucoma Staging System diagram.

The 6 curves dividing OCT Glaucoma Staging System diagram in 6 stages of RNFL thickness damage severity in addition to a normal stage can be expressed by the following non-linear Equation: $\alpha x+\beta x^{2}+\gamma$. Table 1 lists the estimates of $\alpha, \beta$ and $\gamma$ for each of the 6 curves that divides the stages of severity for a patient age range of 60-69 years. 
Table 1 Results of the non-linear equation estimate expressing the separation curves among the OCT Glaucoma Staging System stages for the age range of 60-69 years (asymptotic standard errors in brackets)

\begin{tabular}{lcccc}
\hline Separation curves among stages & $\alpha$ & $\beta$ & $\gamma$ & RMSE / SCTS \\
\hline From stage 0 to borderline & $9.60(1.80)$ & $0.04(0.01)$ & $701.38(105.35)$ & $25.42 / 2479.20$ \\
From borderline to stage 1 & $7.22(1.32)$ & $0.03(0.01)$ & $572.10(72.62)$ & $411.94(48.68)$ \\
From stage 1 to stage 2 & $5.64(0.93)$ & $0.02(0.00)$ & $330.17(33.75)$ & $62.43 / 7664.93$ \\
From stage 2 to stage 3 & $4.56(0.69)$ & $0.02(0.00)$ & $270.96(24.28)$ & $86.86 / 11944.62$ \\
From stage 3 to stage 4 & $3.87(0.54)$ & $0.02(0.00)$ & $229.31(17.70)$ & $112.00 / 17769.27$ \\
From stage 4 to stage 5 & $3.48(042)$ & $0.02(0.00)$ & $140.10 / 26069.93$ \\
\hline
\end{tabular}

Abbreviations: RMSE, root mean square error; SCTS, sum of the corrected total squares.

The two regression lines used to divide defects according to location (superior, inferior, and diffuse) are expressed by the following formulas (for a patient age range of 60-69 years):

- From superior defect to diffuse defect: $y=1.32 \times-$ 40.92 (a)

- From diffuse defect to inferior defect: $y=0.57 x+$ 45.51 (b)

The first regression line (a) separates the defects located in the superior quadrant from defects that involve both the superior and inferior quadrants, considered as diffuse, and located in the central portion of the graph. The second line (b) separates the defects located in the inferior quadrant, from diffuse defects. The classification of defect localization is based on the following assumptions: if the observed $y$ value is lower than the $x$ value by applying the formula ' $2 \mathrm{a}$ ', then the defect is labeled as 'superior defect'; if it is higher than the $x$ value calculated using the formula ' $2 \mathrm{a}$ ', but lower than the value estimated using the formula ' $2 b$ ', then the defect is classified as 'diffuse defect'; if it is higher than the $x$ value obtained using the formula ' $2 \mathrm{~b}$ ', then the defect is considered as 'inferior defect'.

The cohort of 126 subjects, used to assess the sensitivity and specificity of the OCT GSS, included 64 eyes from 64 patients with early OAG (age 64.8 \pm 11.9 ; range 35-82; mean SAP-MD $-2.3 \mathrm{~dB} \pm 0.9$; range -1.6 to $-4.7 \mathrm{~dB}$ ), and 62 eyes from 62 normal subjects (age 61.8 \pm 13.4 ; range 32-84; mean SAP-MD $0.7 \mathrm{~dB} \pm 1.1$ ).

The OCT Glaucoma Staging System correctly classified as normal (stage 0) 57 tests of the 62 healthy subjects. Four were classified as borderline, and one as abnormal; thus providing a specificity ranging between 91.9 and $98.4 \%$, depending on whether borderline results were considered as abnormal or normal, respectively. With regards to the 64 eyes with early glaucoma, one result was excluded due to a clear artifact. Fifty four of the remaining 63 cases were classified as abnormal, 6 as borderline, and 3 as normal (false positives), giving rise to a sensitivity ranging from of 85.7 to $95.2 \%$, depending on the classification of borderline results.

\section{Discussion}

Spectral domain OCT has widely been used to examine the macula and is currently becoming of larger use in managing glaucoma. RNFL measurement by sd-OCTs provide useful information for differentiating patients with $\mathrm{OH}$ without any type of damage from those patients that do not show any functional damage, yet have early structural glaucomatous loss. ${ }^{21,22}$ Moreover, the RNFL assessment can be used to weight the amount of structural loss in glaucoma. For this purpose, a simple and standardized classification of the RNFL damage could prove to be very useful, especially in a day-to-day clinical setting. The GDx Staging System, introduced some years ago and based on data obtained with the GDx VCC, ${ }^{16}$ however, did not take the aging effect on the RNFL thickness into consideration; moreover, the GDx instrument is no longer available on the market. The effect of age on the RNFL thickness is well known, even if the amount of physiological loss is rather different among various studies. ${ }^{23,24}$ As previously demonstrated, the age-related decline in RNFL occurs mainly superiorly, and this trend increases after the age of $50.24,25$

In order to create a classification method using OCT data, we attempted to use several other parameters, however the best ones to stage RNFL damage severity appeared to be the superior and inferior quadrant average, which tends to be directly influenced by glaucomatous damage even in the early stages. These parameters can also be used for indicating the predominant defect location. It is important to stress that RNFL defects often occur in both superior and inferior quadrants, even if the loss is asymmetric. In these cases, the OCT Glaucoma Staging System classifies the defect in the middle part of the graph ('diffuse defect'), or near the edges of this sector. The farther the test results lie from the regression lines, the more probable is that only one quadrant is involved.

A new software was created in order to simplify the classification task: the user has only to insert the type of OCT used, the patient's age, the date of the 
test, the eye tested, and the values of the Superior and Inferior Quadrant Average.

The software (available on the web at the link www.octstagingsystem.com) automatically calculates the severity stage and the location of the defect.

The reliability of the OCT Glaucoma Staging System to correctly stage tests arising from different OCT instruments, of course, needs to be tested in future studies, which are currently ongoing; however, our preliminary data seem to demonstrate that this classification method provides comparable results amongst different instruments.

The sensitivity and specificity of the new classification system in our sample, which included only patients with early glaucoma, was excellent. These results of

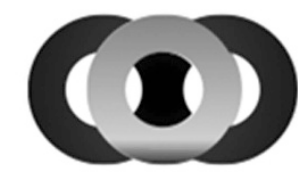

OCT

Glaucoma

Staging System

(๑ Dott. P. Brusini, 2015)

\section{Retinal Nerve Fiber Layer Damage Classification}

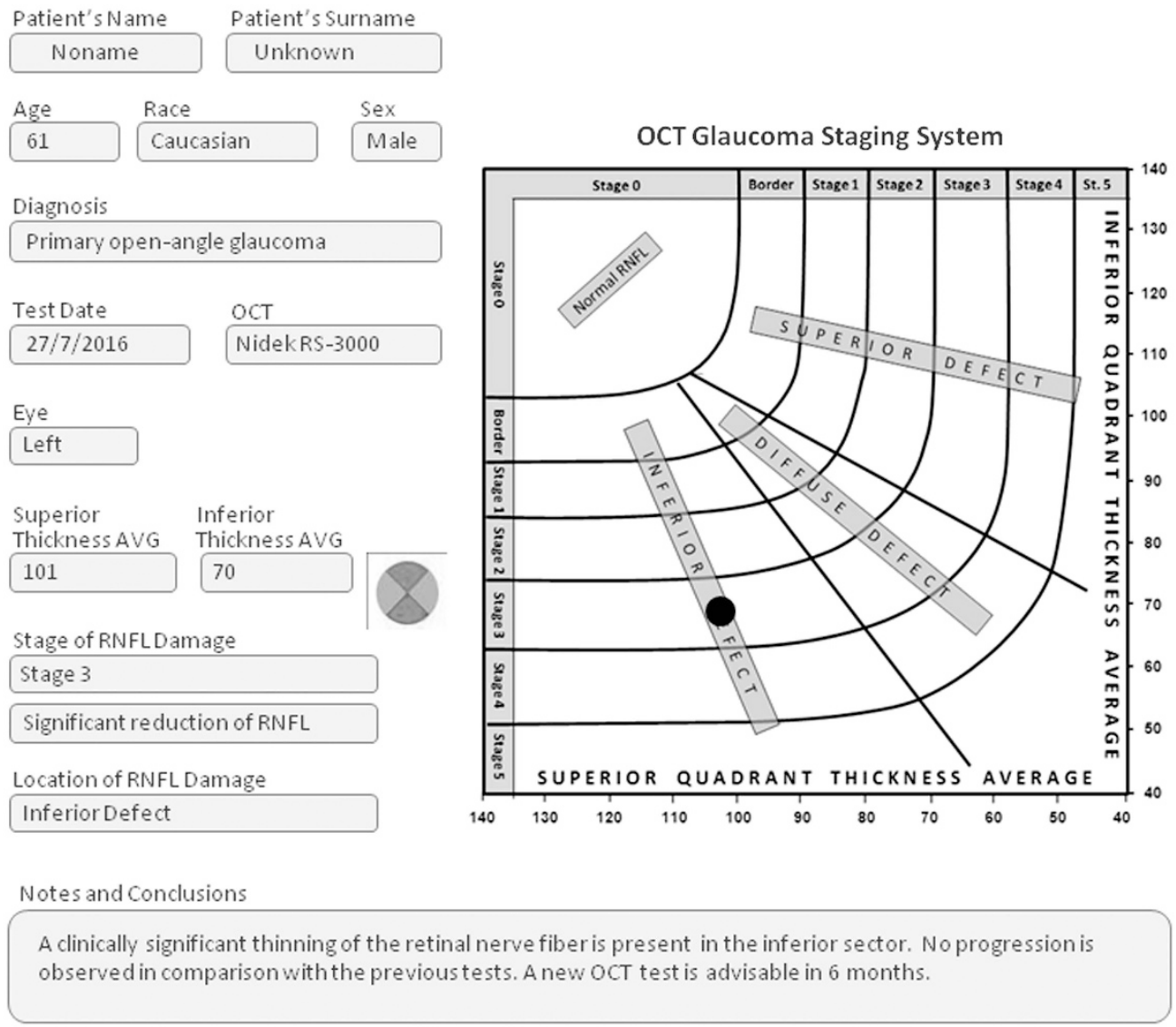

Signature

N.B. This classification system is based on statistics. The interpretation and conclusions are responsability of the clinician.

Figure 2 Printed report from the OCT GSS software (same patient of Figure 1). 
course need to be confirmed by other independent groups.

The OCT Glaucoma Staging System has several limitations, especially considering that there are currently no gold standard methods to compare it with. Future studies using other reference methods are needed to draw definitive conclusions. We used an arbitrary subdivision of the glaucomatous damage severity, which was based on functional and structural loss in an independent cohort. This approach can be biased by several factors, especially considering that SAP is a psycho-physical test, and that the optic disc evaluation was based on a subjective clinical assessment. Moreover, it should be remembered that several artifacts can affect both OCT and SAP results, making the interpretation of data quite difficult with both techniques.

Another possible limitation is that some RNFL slight defects, only shown in the clock-hour map, may go undetected if they do not significantly reduce the quadrant average value. The approach we used, however, should theoretically be more robust against artifacts that can only affect very subtle RNFL sectors.

In the present study, the range of refractive errors of participants was limited, so this system could not be directly applicable to high myopic or hyperopic eyes. Moreover, the peaks of RNFL thickness may be shifted in individuals with high refractive errors, which can alter the results of this classification method.

An additional theoretical drawback is the fact that this system does not take the nasal and temporal sectors into consideration, which are usually not affected by glaucoma damage, even at end stages of the disease.

With these limitations and cautions in mind, we believe the OCT Glaucoma Staging System could be a useful tool in the interpretation of RNFL thickness loss measured with OCT, especially for non expert ophthalmologists, which can obtain an objective, standardized and rapid estimate of structural damage in patients affected by chronic glaucoma. The new software permits a clear written report regarding OCT test results for both patients and clinicians, in addition to further specific notes if needed (Figure 2). This method could also be used for research purposes, making the inclusion criteria of patients in multicenter clinical studies more homogeneous and comparable. Moreover, a standardized classification of the severity of glaucomatous damage may be interesting in deciding on quantity and type of treatment, which should be more aggressive as severity increases. Finally, a classification system with a sufficient number of stages could also prove to be useful in the follow-up assessment, alerting about a possible progression of the glaucomatous damage if a transition in stage occurs.

\section{Summary}

What was known before

- The modern Optical Coherence Tomography (OCTs) supply important and useful information regarding the structural damage in glaucoma. However, currently there is no standardized methods to quantify the Retinal Nerve Fiber Layer (RNFL) damage.

- The current OCT software, based on normative database, only indicate if the RNFL is normal, borderline, or abnormal.

- The user have to decide by his-self how severe the damage is and where it is localized looking at various maps available in the printout.

What this study adds

- The OCT Glaucoma Staging System (OCT GSS) allows the users to stage the RNFL severity damage in a standardized manner.

- It supplies objective information both on the severity of glaucomatous structural loss and on the location of RNFL defects.

- With the new OCT GSS software a reliable quantification of the damage assessed with different types of OCT it is very easy and no time-consuming.

\section{Conflict of interest}

The author declares no conflict of interest.

\section{Acknowledgements}

We would like to thank Dr Maria Letizia Salvetat for her valuable help in statistical analysis of data and Dr Marco Zeppieri for editing this paper. Special thanks to $\mathrm{Mr}$ Guido Battarra and Mr Cristiano Balista for their contribution in the creation of the OCT Glaucoma Staging System software.

\section{References}

1 Schuman JS, Hee MR, Puliafito CA, Wong C, Pedut-Kloizman T, Lin CP et al. Quantification of nerve fiber layer thickness in normal and glaucomatous eyes using optical coherence tomography. Arch Ophthalmol 1995; 113: 586-596.

2 Pieroth L, Schuman JS, Hertzmark E, Hee MR, Wilkins JR, Coker J et al. Evaluation of focal defects of the nerve fiber layer using optical coherence tomography. Ophthalmology 1999; 106: 570-579.

3 Hoh ST, Greenfield DS, Mistlberger A, Liebmann JM, Ishikawa H, Ritch R. Optical coherence tomography and scanning laser polarimetry in normal, ocular hypertensive, and glaucomatous eyes. Am J Ophthalmol 2000; 129: 129-135.

4 Park SB, Sung KR, Kang SY, Kim KR, Kook MS. Comparison of glaucoma diagnostic Capabilities of Cirrus HD and Stratus optical coherence tomography. Arch Ophthalmol 2009; 127: 1603-1609. 
5 Chang RT, Knight OJ, Feuer WJ, Budenz DL. Sensitivity and specificity of time-domain versus spectral-domain optical coherence tomography in diagnosing early to moderate glaucoma. Ophthalmology 2009; 116: 2294-2299.

6 Sung KR, Kim DY, Park SB, Kook MS. Comparison of retinal nerve fiber layer thickness measured by Cirrus HD and Stratus optical coherence tomography. Ophthalmology 2009; 116: 1264-1270.

7 Bengtsson B, Andersson S, Heijl A. Performance of time-domain and spectral-domain Optical Coherence Tomography for glaucoma screening. Acta Ophthalmol 2012; 90: 310-315.

8 Wu H, De Boer JF, Chen TC. Diagnostic capability of spectral-domain optical coherence tomography for glaucoma. Am J Ophthalmol 2012; 153: 815-826.

9 Huang L, Fan N, Shen X, He J. Comparison of the diagnostic ability of retinal nerve fiber layer thickness measured using time domain and spectral domain optical coherence tomography in primary open angle glaucoma. Eye Sci 2011; 26: 132-137.

10 Töteberg-Harms M, Sturm V, Knecht PB, Funk J, Menke MN. Repeatability of nerve fiber layer thickness measurements in patients with glaucoma and without glaucoma using spectral-domain and time-domain OCT. Graefes Arch Clin Exp Ophthalmol 2012; 250: 279-287.

11 Ghasia FF, El-Dairi M, Freedman SF, Rajani A, Asrani S. Reproducibility of spectral-domain optical coherence tomography measurements in adult and pediatric glaucoma. J Glaucoma 2015; 24: 55-63.

12 Cremasco F, Massa G, Gonçalves Vidotti V, Pedroso de Carvalho Lupinacci Á, Costa VP. Intrasession, intersession, and interexaminer variabilities of retinal nerve fiber layer measurements with spectral-domain OCT. Eur J Ophthalmol 2011; 21: 264-270.

$13 \mathrm{Wu} \mathrm{H}$, de Boer JF, Chen TC. Reproducibility of retinal nerve fiber layer thickness measurements using spectral domain optical coherence tomography. J Glaucoma 2011; 20: 470-476.

14 Lee SH, Kim SH, Kim TW, Park KH, Kim DM.

Reproducibility of retinal nerve fiber thickness measurements using the test-retest function of spectral OCT/SLO in normal and glaucomatous eyes. J Glaucoma 2010; 19: 637-642.
15 Brusini P, Johnson CA. Staging functional damage in glaucoma: review of different classification methods. Surv Ophthalmol 2007; 52: 156-179.

16 Brusini P. GDx Staging System: a new method for retinal nerve fiber layer damage classification. J Glaucoma 2011; 20: 287-293.

17 Anderson D, Patella V. Automated Static Perimetry. 2nd edn Mosby. Inc: St Louis, MO, USA, 1999.

18 Brusini P, Filacorda S. Enhanced Glaucoma Staging System (GSS 2) for classifying functional damage in glaucoma. J Glaucoma 2006; 15: 40-46.

19 Pierro L, Gagliardi M, Iuliano L, Ambrosi A, Bandello F. Retinal nerve fiber layer thickness reproducibility using seven different OCT instruments. Invest Ophthalmol Vis Sci 2012; 53: 5912-5920.

20 Brusini P, Zeppieri M, Tosoni C, Parisi L, Salvetat ML. Optic Disc Damage Staging System (ODDSS). J Glaucoma 2010; 19: 442-449.

21 Lisboa R, Leite MT, Zangwill LM, Tafreshi A, Weinreb RN, Medeiros FA. Diagnosing preperimetric glaucoma with spectral domain optical coherence tomography. Ophthalmology 2012; 119: 2261-2269.

22 Jeoung JW, Kim TW, Weinreb RN, Kim SH, Park KH, Kim DM. Diagnostic ability of spectral-domain versus timedomain optical coherence tomography in preperimetric glaucoma. J Glaucoma 2014; 23: 299-306.

23 Sung KR, Wollstein G, Bilonick RA, Townsend KA, Ishikawa $\mathrm{H}$, Kagemann $\mathrm{L}$ et al. Effects of age on optical coherence tomography measurements of healthy retinal nerve fiber layer, macula, and optic nerve head. Ophthalmology 2009; 116: 1119-1124.

24 Leung CKS, Yu M, Weinreb RN, Ye C, Liu S, Lai G et al. Retinal nerve fiber layer imaging with spectral-domain optical coherence tomography: a prospective analysis of age-related loss. Ophthalmology 2012; 119: 731-737.

25 Feuer WJ, Budenz DL, Anderson DR, Cantor L, Greenfield DS, Savell J et al. Topographic differences in the age-related changes in the retinal nerve fiber layer of normal eyes measured by Stratus optical coherence tomography. J Glaucoma 2011; 20: 133-138. 